\title{
TOPOLOGICALLY PRINCIPAL PART OF ANALYTIC FUNCTIONS
}

\author{
ETSUO YOSHINAGA
}

Dedicated to Professor Yukihiro Kodama on his 60th birthday

\begin{abstract}
The problem of $C^{0}$-sufficiency of jets is one of the most interesting problems in the theory of functions or singularities. Roughly speaking, it is the problem of determining a topologically principal part of the Taylor expansion of a given function $f(x)$ at the origin of Euclidean space. Here, the topologically principal part should satisfy the properties that it is as small as possible a part of the Taylor expansion of $f(x)$ and that the local topological type of $f(x)$ at the origin is determined by it. If a function $f(x)$ is an isolated singularity at the origin or has a nondegenerate Newton principal part (see (1.2)), then we know some answers to this problem (see (1.1), (1.3)). The purpose of this paper is to give some results for this problem for any analytic function. The main results are formulated in (1.5), (1.6), and (1.7).
\end{abstract}

\section{Main Results}

Let $K:=\mathbf{R}$ or $\mathbf{C}$ and $A\left(K^{n}\right)$ be the set of all germs of analytic functions $f:\left(K^{n}, 0\right) \rightarrow(K, 0)$ at the origin of $K^{n}$.

Sufficiency of $f \in A\left(K^{n}\right)$ with an isolated singularity at the origin of $K^{n}$ has been studied by many mathematicians [1, 3, 4]. Kuiper, Kuo, Chang and Lu proved the following:

(1.1) Theorem [1, 3, 4]. Let $f \in A\left(K^{n}\right)$ be an isolated singularity. If there exist positive $\varepsilon$ and $\delta$ such that

$$
\left|\left(\frac{\partial f}{\partial x_{1}}, \frac{\partial f}{\partial x_{2}}, \ldots, \frac{\partial f}{\partial x_{n}}\right)\right| \geq \varepsilon|x|^{r-\delta}
$$

for some positive integer $r$ and for all $x$ in a neighborhood of the origin of $K^{n}$, then the r-jet $j^{(r)}(f)$ is a $C^{0}$-sufficient jet.

Let $\Gamma_{+}(f)$ be the Newton polygon of $f \in A\left(K^{n}\right)$, the convex hull of the set

$$
\bigcup\left\{k+\mathbf{R}_{+}^{n} \mid a_{k} \neq 0\right\}
$$

Received by the editors October 2, 1987 and, in revised form, February 24, 1988.

1980 Mathematics Subject Classification (1985 Revision). Primary 58C28; Secondary 57R52.

Key words and phrases. Topologically principal part, topological triviality, nondegenerate Newton principal part. 
in $\mathbf{R}^{n}$ where $\mathbf{R}_{+}$is the set of all nonnegative real numbers, for the Taylor expansion

$$
f(x)=\sum_{k} a_{k} x^{k}=\sum_{k} a_{k_{1}, k_{2}, \ldots, k_{n}} x_{1}^{k_{1}} x_{2}^{k_{2}} \cdots x_{n}^{k_{n}} .
$$

Let $S$ be a subset of $\mathbf{Z}_{+}^{n}$ (or $\mathbf{R}_{+}^{n}$ ) where $\mathbf{Z}_{+}$is the set of all nonnegative integers and define $f_{S}:=f \mid S:=\sum_{k \in S \cap \mathbf{Z}_{+}^{n}} a_{k} x^{k}$.

(1.2) Definition. An $f \in A\left(K^{n}\right)$ (or the Newton principal part of $f$ ) is nondegenerate if $\left\{x \in K^{n} \mid \partial f_{\gamma} / \partial x_{1}=\partial f_{\gamma} / \partial x_{2}=\cdots=\partial f_{\gamma} / \partial x_{n}=0\right\} \subset$ $\left\{x_{1} x_{2} \cdots x_{n}=0\right\}$ for any compact face $\gamma$ of $\Gamma_{+}(f)$.

The following theorem related to sufficiency of nondegenerate analytic functions is well known.

(1.3) Theorem [2]. Let an $f \in A\left(K^{n}\right)$ be nondegenerate and $\Gamma_{+}(f)$ be the Newton polygon of $f$. Then the family $f+u g$ is topologically trivial along $I=\{u \in \mathbf{R} \mid 0 \leq u \leq 1\}$ for any $g \in A\left(K^{n}\right)$ with $\Gamma_{+}(g) \subset \operatorname{Int} \Gamma_{+}(f)$.

(1.4) Definitions.

$$
|\operatorname{Grad} f|^{2}:=\left|\left(x_{1} \frac{\partial f}{\partial x_{1}}, x_{2} \frac{\partial f}{\partial x_{2}}, \cdots, x_{n} \frac{\partial f}{\partial x_{n}}\right)\right|^{2}=\sum_{i=1}^{n}\left|x_{i} \frac{\partial f}{\partial x_{i}}\right|^{2} .
$$

For a given $k \in \mathbf{Z}_{+}^{n}$, define

$(1.4 .2)_{k}$ Condition: There exists a positive $\varepsilon=\varepsilon(k)$ such that

$$
|\operatorname{Grad} f| \geq \varepsilon\left|x^{k}\right|
$$

in a neighborhood of the origin of $K^{n}$.

(1.4.3) Let $\Lambda_{+}(f)$ be the convex hull of the set

$$
\bigcup\left\{k+\mathbf{R}_{+}^{n} \mid \text { Condition }(1.4 .2)_{k} \text { holds }\right\}
$$

in $\mathbf{R}^{n}$. We call $\Lambda_{+}(f)$ a gradient polygon of $f$.

Let $m$ be the order of the function $|\operatorname{Grad} f|$ on $V:=V(f):=\left\{x \in K^{n} \mid\right.$ $|\operatorname{Grad} f|=0\}$. Namely

$$
m:=m(f):=\frac{1}{2} \operatorname{Min}\left\{\text { the order of }|\operatorname{Grad} f|^{2} \text { at } x_{0} \mid x_{0} \in V(f)\right\} .
$$

For a given $k \in \mathbf{Z}_{+}^{n}$, define

(1.4.5) ${ }_{k}$ Condition: There exists a positive $\varepsilon=\varepsilon(k)$ such that

$$
|\operatorname{Grad} f|^{1+1 / m} \geq \varepsilon\left|x^{k}\right|
$$

in a neighborhood of the origin of $K^{n}$.

(1.4.6) Let $\tilde{\Lambda}_{+}(f)$ be the convex hull of the set

$$
\bigcup\left\{k+\mathbf{R}_{+}^{n} \mid \text { Condition }(1.4 .5)_{k} \text { holds }\right\}
$$

in $\mathbf{R}^{n}$. We call $\tilde{\Lambda}_{+}(f)$ a quasi gradient polygon of $f$. 
We need the following notation to state Lemma 2.6 and Corollary 2.7.

For a given $k \in \mathbf{Z}_{+}^{n}$, define

$$
\mu(k):=\operatorname{Max}\left\{\mu \in \mathbf{R} \mid \frac{1}{1+\mu} k \in \Lambda_{+}(f)\right\}
$$

and

$$
\mu:=\mu(f):=\operatorname{Min}\left\{\mu(k) \mid k \in \operatorname{Int} \Lambda_{+}(f) \cap \mathbf{Z}^{n}\right\} .
$$

Then $\Gamma_{+}(f) \supset \Lambda_{+}(f) \supset \tilde{\Lambda}_{+}(f)$ where the first inclusion is proved in (3.1) and the second one is clear by definition.

Now the main results in this paper are the following three theorems.

(1.5) Theorem. Suppose that one of the following two conditions holds for an analytic function $g(x)$ :

$$
\begin{gathered}
\Gamma_{+}(g) \subset \tilde{\Lambda}_{+}(f) ; \\
\Gamma_{+}(g) \subset \operatorname{Int} \Lambda_{+}(f) \text { and } V(f)=\{0\} .
\end{gathered}
$$

Then the family $f+u g$ is topologically trivial, identically on $V(f)$, along $I:=$ $\{u \in \mathbf{R} \mid 0 \leq u \leq 1\}$. Namely there exists a local homeomorphism $H:\left(K^{n} \times\right.$ $\mathbf{R}, 0 \times I) \rightarrow\left(K^{n} \times \mathbf{R}, 0 \times I\right)$ such that the following diagram (1.5.3) commutes:

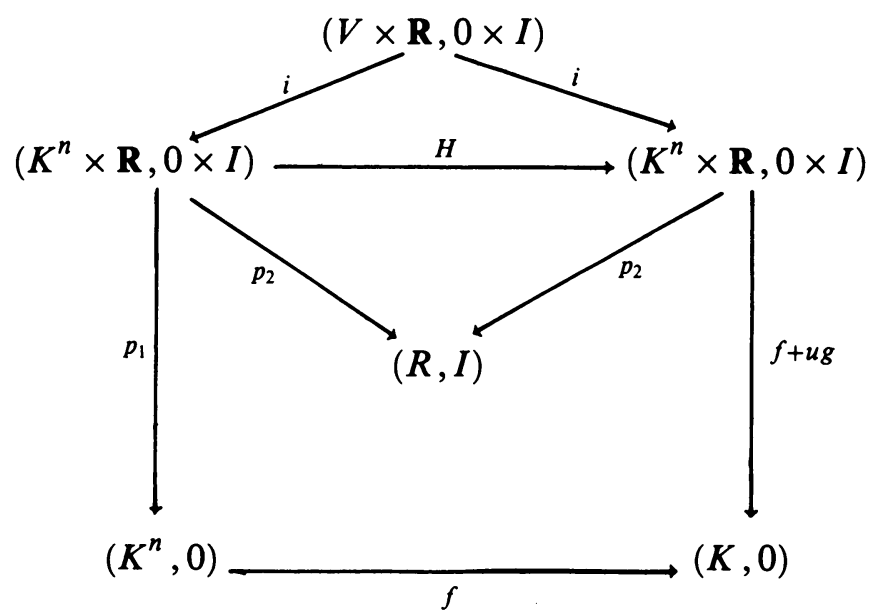

where $p_{1}, p_{2}$ are the canonical projections and $i$ is the inclusion map.

(1.6) Theorem. Suppose $\Gamma_{+}(g) \subset \Lambda_{+}(f)$ for an analytic function $g$ and $V(f)=$ $\{0\}$. Then $f(x)+u g(x)$ is topologically trivial along $I(\delta)=\{u \in \mathbf{R} \mid 0 \leq u \leq \delta\}$ for a sufficiently small positive $\delta$. Namely there exists a local homeomorphism $H:\left(K^{n} \times \mathbf{R}, 0 \times 0\right) \rightarrow\left(K^{n} \times \mathbf{R}, 0 \times 0\right)$ such that the diagram (1.5.3) replacing $I$ by 0 commutes.

(1.7) Theorem. An $f$ is nondegenerate if and only if $\Gamma_{+}(f)=\Lambda_{+}(f)$.

Let $\Lambda_{-}(f)\left(\right.$ resp. $\left.\tilde{\Lambda}_{-}(f)\right)$ be the complement of Int $\Lambda_{+}(f)\left(\right.$ resp. Int $\left.\tilde{\Lambda}_{+}(f)\right)$ in $\mathbf{R}_{+}^{n}$. By (1.5), $f \mid \tilde{\Lambda}_{-}(f)$ (resp. $f \mid \Lambda_{-}(f)$ if $V=\{0\}$ ) is a topologically 
principal part of $f$ in the sense that if $f\left|\tilde{\Lambda}_{-}(f)=g\right| \tilde{\Lambda}_{-}(f)$ (resp. $f \mid$ $\left.\Lambda_{-}(f)=g \mid \Lambda_{-}(f)\right)$ for $f, g \in A\left(K^{n}\right)$; then $f$ is topologically equivalent (identically on $V(f)$ ) to $g$.

Suzuki [5] proved a beautiful theorem related to (1.6) when $f(x, y)$ is a weighted homogeneous polynomial of two complex variables with an isolated singularity.

\section{Proof of Theorems (1.5) AND (1.6)}

For $f, g \in A\left(K^{n}\right)$, we let

$$
\begin{aligned}
F(x ; u) & :=f(x)+u g(x), \\
\operatorname{Grad} F & :=\left(x_{1} \frac{\partial F}{\partial x_{1}}, x_{2} \frac{\partial F}{\partial x_{2}}, \ldots, x_{n} \frac{\partial F}{\partial x_{n}}, \frac{\partial F}{\partial u}\right), \\
\operatorname{Grad}_{x} F & :=\left(x_{1} \frac{\partial F}{\partial x_{1}}, x_{2} \frac{\partial F}{\partial x_{2}}, \ldots, x_{n} \frac{\partial F}{\partial x_{n}}\right), \\
p(x ; u) & :=\bar{g}\left|\operatorname{Grad}_{x} F\right|^{-2}\left(\left|x_{1}\right|^{2} \frac{\partial F}{\partial x_{1}},\left|x_{2}\right|^{2} \frac{\partial F}{\partial x_{2}}, \ldots,\left|x_{n}\right|^{2} \frac{\partial F}{\partial x_{n}}, 0\right), \\
V:=: V(f) & :=\left\{x \in K^{n}|| \operatorname{Grad} f \mid=0\right\},
\end{aligned}
$$

and

$$
X(x ; u):= \begin{cases}(0,1)-\overline{p(x ; u)} & \text { if } x \notin V, \\ (0,1) & \text { otherwise, }\end{cases}
$$

where $\bar{g}$ means the complex conjugate of $g$.

(2.1) Lemma. If $\Gamma_{+}(g) \subset \tilde{\Lambda}_{+}(f)\left(\right.$ resp. $\left.\Gamma_{+}(g) \subset \Lambda_{+}(f)\right)$, then

$$
2\left|\operatorname{Grad}_{x} F\right| \geq|\operatorname{Grad} f|
$$

in a neighborhood of $\{0\} \times I$ (resp. $\{0\} \times\{0\})$ in $K^{n} \times \mathbf{R}$.

Proof. If $\Gamma_{+}(g) \subset \tilde{\Lambda}_{+}(f)$ (resp. $\left.\Gamma_{+}(g) \subset \Lambda_{+}(f)\right)$, then

$$
\Gamma_{+}\left(x_{i} \frac{\partial g}{\partial x_{i}}\right) \subset \tilde{\Lambda}_{+}(f) \quad\left(\text { resp. } \Gamma_{+}\left(x_{i} \frac{\partial g}{\partial x_{i}}\right) \subset \Lambda_{+}(f)\right) .
$$

So the hypothesis of (2.1) implies

$$
\sum_{i=1}^{n}\left|x_{i} \frac{\partial f}{\partial x_{i}}\right|^{2} \geq 4|u|^{2} \sum_{i=1}^{n}\left|x_{i} \frac{\partial g}{\partial x_{i}}\right|^{2}
$$

in a neighborhood of $\{0\} \times I$ (resp. $\{0\} \times\{0\})$ in $K^{n} \times \mathbf{R}$.

Hence,

$$
\begin{aligned}
\left|\operatorname{Grad}_{x} F\right|^{2} & =\sum_{i=1}^{n}\left|x_{i} \frac{\partial F}{\partial x_{i}}\right|^{2}=\sum_{i=1}^{n}\left|x_{i} \frac{\partial f}{\partial x_{i}}+u x_{i} \frac{\partial g}{\partial x_{i}}\right|^{2} \\
& =|\operatorname{Grad} f+u \operatorname{Grad} g|^{2} \geq(|\operatorname{Grad} f|-|u \operatorname{Grad} g|)^{2} \\
& \geq \frac{1}{4}|\operatorname{Grad} f|^{2}
\end{aligned}
$$


in a neighborhood of $\{0\} \times I$ (resp. $\{0\} \times\{0\}$ ). This completes the proof of (2.1).

The following (2.2) is an immediate corollary of (2.1).

(2.2) Corollary. If $\Gamma_{+}(g) \subset \tilde{\Lambda}_{+}(f)$ (resp. $\left.\Gamma_{+}(g) \subset \Lambda_{+}(f)\right)$, then $\{(x, u) \in$ $\left.K^{n} \times I|| \operatorname{Grad}_{x} F \mid=0\right\} \subset V \times I$ as germs at $\{0\} \times I$ (resp. $\left.\{0\} \times\{0\}\right)$ where $I=[0,1]$, and so the vector field $X$ is well defined in a neighborhood of $\{0\} \times I$ (resp. $\{0\} \times\{0\})$.

\section{(2.3) Lemma.}

(2.3.1) Suppose that (1.5.1) holds. Then there exists a positive $K$ such that

$$
\left|X(x ; u)-X\left(x_{0} ; u\right)\right| \leq K\left|x-x_{0}\right|
$$

for any $x_{0} \in V$, in a neighborhood of $\{0\} \times I$ in $K^{n} \times \mathbf{R}$.

(2.3.2) If $\Gamma_{+}(g) \subset$ Int $\Lambda_{+}(f)$ (resp. $\left.\Gamma_{+}(g) \subset \Lambda_{+}(f)\right)$, then there exists a positive $K$ such that

$$
\left|X(x ; u)-X\left(x_{0} ; u\right)\right| \leq K|x|
$$

for any $x_{0} \in V$, in a neighborhood of $\{0\} \times I$ (resp. $\left.\{0\} \times\{0\}\right)$ in $K^{n} \times \mathbf{R}$.

Proof. If $x \in V$, then (2.3) is trivial. So we assume $x \notin V$. Then we have

$$
\begin{aligned}
\left|X(x ; u)-X\left(x_{0} ; u\right)\right| & =|p(x ; u)| \\
& =\left|g \| \operatorname{Grad}_{x} F\right|^{-2}\left(\sum_{i=1}^{n}\left|x_{i}^{4}\right|\left|\frac{\partial F}{\partial x_{i}}\right|^{2}\right)^{1 / 2} \\
& \leq\left|g \| \operatorname{Grad}_{x} F\right|^{-1}|x| \\
& \leq 2\left|g \| \operatorname{Grad}^{-1}\right| x \mid
\end{aligned}
$$

in a neighborhood of $\{0\} \times I$ (resp. $\{0\} \times\{0\})$ if $\Gamma_{+}(g) \subset \tilde{\Lambda}_{+}(f)$ (resp. $\left.\Gamma_{+}(g) \subset \Lambda_{+}(f)\right)$, where the last inequality follows from $(2.1)$.

If (1.5.1) holds, then there exist positive $\varepsilon, K$ such that

$$
2|g||\operatorname{Grad} f|^{-1} \leq \frac{1}{\varepsilon}|\operatorname{Grad} f|^{1 / m} \leq K\left|x-x_{0}\right|
$$

in a neighborhood of the origin. This completes the proof of (2.3.1).

If $\Gamma_{+}(g) \subset$ Int $\Lambda_{+}(f)$ (resp. $\left.\Gamma_{+}(g) \subset \Lambda_{+}(f)\right)$, there exists a positive $K$ such that

$$
2|g||\operatorname{Grad} f|^{-1}|x| \leq K|x|
$$

in a neighborhood of the origin. This completes the proof of (2.3.2).

The conclusion of (2.3) is a well-known Lipschitz condition for the existence of a locally unique solution of an ordinary differential equation (see [1, p. 876]). So we have the following corollary. 
(2.4) Corollary. Suppose that one of (1.5.1), (1.5.2) holds (resp. $\Gamma_{+}(g) \subset$ $\left.\Lambda_{+}(f)\right)$. Then the vector field $X(x ; u)$ is continuous in a neighborhood of $\{0\} \times I$ (resp. $\{0\} \times\{0\})$ and the differential equation

$$
\left(\frac{d x}{d t}, \frac{d u}{d t}\right)=X(x ; u)
$$

has a locally unique continuous solution $\phi(t ; x, u)$ with $\phi(0 ; x, u)=(x, u)$ in a neighborhood of $\{0\} \times I$ (resp. $\{0\} \times\{0\})$.

(2.5) Lemma. The function $F(\phi(t ; x, u))$ of $t$ is constant along each solution curve $\phi$ of (2.4.1) under the assumption of (1.5) or (1.6).

Proof. If $x \notin V$, then

$$
\begin{aligned}
\frac{d}{d t} F(\phi(t ; x, u)) & =\frac{\partial F}{\partial x_{1}} \frac{d \phi_{1}}{d t}+\frac{\partial F}{\partial x_{2}} \frac{d \phi_{2}}{d t}+\cdots+\frac{\partial F}{\partial x_{n}} \frac{d \phi_{n}}{d t}+\frac{\partial F}{\partial u} \\
& =\left\langle\left(\frac{\partial F}{\partial x_{1}}, \frac{\partial F}{\partial x_{2}}, \ldots, \frac{\partial F}{\partial x_{n}}, \frac{\partial F}{\partial u}\right), \overline{\left(\frac{d \phi}{d t}\right)}\right\rangle \\
& =\left\langle\left(\frac{\partial F}{\partial x_{1}}, \frac{\partial F}{\partial x_{2}}, \ldots, \frac{\partial F}{\partial x_{n}}, \frac{\partial F}{\partial u}\right), \overline{X(x ; u)}\right\rangle \\
& =\frac{\partial F}{\partial u}-g\left|\operatorname{Grad}_{x} F\right|^{-2} \sum_{i=1}^{n}\left|x_{i} \frac{\partial F}{\partial x_{i}}\right|^{2} \\
& =\frac{\partial F}{\partial u}-g \\
& =0
\end{aligned}
$$

for all $t \geq 0$. If $x_{0} \in V$, then

$$
\frac{d}{d t} F(\phi(t))=g\left(x_{0}\right)=0, \quad t \geq 0,
$$

also holds, since $F(\phi(t))=F\left(x_{0} ; u+t\right)=f\left(x_{0}\right)+(u+t) g\left(x_{0}\right)$ and $\left|\operatorname{Grad}_{x} f\right| \geq$ $\varepsilon|g|$ by the common assumption $\Gamma_{+}(g) \subset \Lambda_{+}(f)$ of (1.5) and (1.6). We thus draw the conclusion that $F(\phi(t))=$ constant along each solution curve $\phi$ of (2.4.1).

Now, we are at the final stage of the proof of (1.5) and (1.6). Under the assumption of (1.5) (resp. (1.6)), the differential equation (2.4.1) has a locally unique continuous solution $\phi(t ; x, u)$ by (2.5). Since $I$ is compact and the $u$-component of $X(x ; u)$ is equal to one, a finite succession of solutions gives the trajectory starting from $(x, 0)$ in a neighborhood of $(0,0)$, ending at a point of $K^{n} \times\{1\}$. Namely, denoting the trajectory starting from $(x, 0)$ by $\phi(u ; x, 0)$, we have

$$
\begin{aligned}
H(x ; u) & :=\phi(u ; x, 0):\left(K^{n} \times \mathbf{R}, 0 \times I\right) \rightarrow\left(K^{n} \times \mathbf{R}, 0 \times I\right) \\
& \left(\text { resp. } H(x ; u):=\phi(u ; x, 0):\left(K^{n} \times \mathbf{R}, 0 \times 0\right) \rightarrow\left(K^{n} \times \mathbf{R}, 0 \times 0\right)\right),
\end{aligned}
$$

a level preserving local homeomorphism, identically on $V \times I$. In fact, the uniqueness of solutions of (2.4.1) implies that $H$ is locally homeomorphic. 
And the diagram (1.5.3) (resp. (1.5.3) replacing $I$ by 0$)$ commutes. This completes the proofs of (1.5) and (1.6).

The method used here to prove (1.5) and (1.6) modifies a method used in $[1,4]$. One of the most important improvements is the definition of the vector field $X(x ; u)$. The definition of "gradient vector" Grad $F$ used here has some reasonable meaning in the sense of a "singular" Riemannian metric (see $[2,7])$.

(2.6) Lemma. If $1 \leq m(f) \mu(f)$, then Int $\Lambda_{+}(f) \subset \tilde{\Lambda}_{+}(f)$.

Proof. By the definition (1.4.7) of $\mu(f)$, we have

$$
\frac{1}{1+\mu} k \in \Lambda_{+}(f)
$$

for any $k \in \operatorname{Int} \Lambda_{+}(f)$. So there exists a positive $\varepsilon$ such that

$$
\varepsilon|x|^{k} \leq|\operatorname{Grad} f|^{1+\mu} \leq|\operatorname{Grad} f|^{1+1 / m}
$$

in a neighborhood of the origin of $K^{n}$. Thus $k \in \tilde{\Lambda}_{+}(f)$. This completes the proof of (2.6).

The following (2.7) is an immediate corollary of (1.5) and (2.6).

(2.7) Corollary. Suppose $1 \leq m(f) \mu(f)$. Then for any $g \in A\left(K^{n}\right)$, the family $f+u g$ is topologically trivial, identically on $V(f)$, along $I=\{0 \leq u \leq 1\}$ if $\Gamma_{+}(g) \subset \operatorname{Int} \Lambda_{+}(f)$.

(2.8) Example. Let $f(x, y):=(x+y)^{2}+y^{3}$ and $g(x, y):=-y^{3}$. Then $\Gamma_{+}(g) \subset \operatorname{Int} \Gamma_{+}(f)$ and $V(f)=\{0\}$ as germs at the origin. But it is clear that the family $F(x, y ; u):=f+u g$ is not topologically trivial along $I=[0,1]$ because $F(x, y ; 0)=f(x, y)$ is not locally topological equivalent to $F(x, y ; 1)=$ $(x+y)^{2}$.

Example (2.8) shows that we may not in general use the Newton polygon $\Gamma_{+}(f)$ instead of the gradient polygon $\Lambda_{+}(f)$ in (1.5.2).

\section{Proof of (1.7)}

At the beginning of this section we recall the notion of toroidal embedding corresponding to the Newton polygon $\Gamma_{+}(f)$ of $f \in A\left(K^{n}\right)$. It plays an essential role in the proof of Theorem (1.6).

Let $\mathbf{R}^{n *}$ be the dual space of $\mathbf{R}^{n}$. For $a=\left(a_{1}, a_{2}, \ldots, a_{n}\right) \in \mathbf{R}_{+}^{n}$ and $\alpha=\left(\alpha_{1}, \alpha_{2}, \ldots, \alpha_{n}\right) \in \mathbf{R}_{+}^{n *}$, define:

$$
\begin{aligned}
\langle a, \alpha\rangle & :=a_{1} \alpha_{1}+a_{2} \alpha_{2}+\cdots+a_{n} \alpha_{n}, \\
l(\alpha) & :=\min \left\{\langle a, \alpha\rangle \mid a \in \Gamma_{+}(f)\right\}, \\
\gamma(\alpha) & :=\left\{a \in \Gamma_{+}(f) \mid\langle a, \alpha\rangle=l(\alpha)\right\},
\end{aligned}
$$

and

$$
\Gamma^{*}(f):=\mathbf{R}_{+}^{n *} / \sim,
$$


where $\alpha \sim \alpha^{\prime}$ defined by $\gamma(\alpha)=\gamma\left(\alpha^{\prime}\right)$. We can identify an equivalence class $\sigma$ with a rational polyhedral convex cone

$$
\mathbf{R}_{+} a^{1}(\sigma)+\mathbf{R}_{+} a^{2}(\sigma)+\cdots+\mathbf{R}_{+} a^{n}(\sigma)
$$

where $a^{1}(\sigma), a^{2}(\sigma), \ldots, a^{n}(\sigma)$ are minimal generators and we require $a^{i}(\sigma) \epsilon$ $\mathbf{Z}_{+}^{n}$ to be as small as possible. Then there exists a unimodular simplicial subdivision $\Sigma$ of $\Gamma^{*}(f)$; namely $\Sigma$ is a simplicial subdivision of $\Gamma^{*}(f)$ and $\operatorname{det}\left(a^{1}(\sigma), a^{2}(\sigma), \ldots, a^{n}(\sigma)\right)= \pm 1$ for any $n$-dimensional simplex $\sigma$ of $\Sigma$ [6].

Let $K^{n}(\sigma)$ be a copy of $K^{n}$ for any $n$-dimensional simplex $\sigma \in \Sigma$ and $\left(y_{\sigma, 1}, y_{\sigma, 2}, \ldots, y_{\sigma, n}\right)$ be a coordinate system of $K^{n}(\sigma)$.

For a matrix $A=\left(a_{i}^{j}\right) \in \operatorname{Mat}(n \times n, \mathbf{Z})$, define

$$
A_{y:=\left(y_{1}^{a_{1}^{1}} y_{2}^{a_{1}^{2}} \ldots y_{n}^{a_{1}^{n}}, \ldots, y_{1}^{a_{n}^{1}} y_{2}^{a_{n}^{2}} \ldots y_{n}^{a_{n}^{n}}\right)}
$$

Let

$$
a(\sigma):=\left(a^{1}(\sigma), a^{2}(\sigma), \ldots, a^{n}(\sigma)\right)
$$

and

$$
a^{i}(\sigma)={ }^{t}\left(a_{1}^{i}(\sigma), a_{2}^{i}(\sigma), \ldots, a_{n}^{i}(\sigma)\right) .
$$

Define

$$
X:=X\left(\Gamma_{+}(f)\right):=\bigcup_{\sigma} K^{n}(\sigma) / \sim
$$

where $y_{\sigma} \sim y_{\tau} \quad\left(y_{\sigma} \in K^{n}(\sigma), y_{\tau} \in K^{n}(\tau)\right)$ is defined by $y_{\tau}={ }^{a(\tau)^{-1} a(\sigma)} y_{\sigma}$. Then $X$ is a nonsingular algebraic manifold and the mapping

$$
\pi: X \rightarrow K^{n}, \quad \pi\left(y_{\sigma}\right)={ }^{a(\sigma)} y_{\sigma} \quad\left(y_{\sigma} \in K^{n}(\sigma)\right)
$$

is a proper analytic mapping onto $K^{n}$.

Put

$$
\pi_{\sigma}:=\pi \mid K^{n}(\sigma): K^{n}(\sigma) \rightarrow K^{n}
$$

and

$$
g_{i}\left(y_{\sigma}\right):=\frac{\left(x_{i} \partial f / \partial x_{i}\right) \circ \pi_{\sigma}\left(y_{\sigma}\right)}{\prod_{j=1}^{n} y_{\sigma, j}^{((a j)(\sigma))}},
$$

an analytic function in $K^{n}(\sigma)$. Then we have the following

(3.1) Lemma. $\Lambda_{+}(f) \subset \Gamma_{+}(f)$.

Proof. Let $k=\left(k_{1}, k_{2}, \ldots, k_{n}\right)$ be an element of $\mathbf{Z}_{+}^{n}-\Gamma_{+}(f)$. Then there exist an $n$-dimensional simplex $\sigma$ of $\Sigma$ and $i_{0}$ such that

$$
l\left(a^{i_{0}}(\sigma)\right)>\left\langle k, a^{i_{0}}\right\rangle .
$$

Then we have

$$
|\operatorname{Grad} f| \circ \pi_{\sigma}\left(y_{\sigma}\right)=\left|y_{1}^{l\left(a^{\prime}(\sigma)\right)} y_{2}^{l\left(a^{2}(\sigma)\right)} \cdots y_{n}^{l\left(a^{n}(\sigma)\right)}\right|\left(\sum_{i=1}^{n}\left|g_{i}\left(y_{\sigma}\right)\right|^{2}\right)^{1 / 2}
$$


and

$$
\left|x^{k}\right| \circ \pi_{\sigma}\left(y_{\sigma}\right)=\prod_{i=1}^{n}\left|x_{i}^{k_{i}}\right| \circ \pi_{\sigma}\left(y_{\sigma}\right)=\left|y_{1}^{K_{1}} y_{2}^{K_{2}} \cdots y_{n}^{K_{n}}\right|
$$

where $K_{i}=\sum_{j=1}^{n} k_{j} a_{j}^{i}(\sigma)=\left\langle k, a^{i}(\sigma)\right\rangle$. This shows that the inequality

$$
|\operatorname{Grad} f| \circ \pi_{\sigma}(y)_{\sigma} \geq \varepsilon\left|x^{k}\right| \circ \pi_{\sigma}\left(y_{\sigma}\right)
$$

does not hold in any neighborhood of the origin of $K^{n}(\sigma)$ for any positive $\varepsilon$. So $k$ is not the element of $\Lambda_{+}(f)$. This completes the proof of (3.1).

(3.2) Lemma. A germ $f$ is nondegenerate if and only if $\sum_{i=1}^{n}\left|g_{i}\left(y_{\sigma}\right)\right|^{2} \neq 0$ for any $y_{\sigma} \in \pi_{\sigma}^{-1}(0)$ and any n-dimensional simplex $\sigma$ of $\Sigma$.

Proof. The "only if" part of (3.2) is Lemma 3.9 in [2, p. 472].

Suppose that $f$ is degenerate. Then there exist a compact face $\gamma$ of $\Gamma_{+}(f)$ and a point $x^{0}$ of $\left(K^{*}\right)^{n}$ such that

$$
\left(x_{i} \partial f_{\gamma} / \partial x_{i}\right)\left(x^{0}\right)=0, \quad 1 \leq i \leq n .
$$

There exist an $n$-dimensional simplex $\sigma$ of $\Sigma$ and a subset $I$ of $\{1,2, \ldots, n\}$ such that

$$
\gamma=\bigcap_{i \in I} \gamma\left(a^{i}(\sigma)\right)
$$

Define $E_{\sigma, I}:=\left\{y_{\sigma} \in K^{n}(\sigma) \mid y_{\sigma, i}=0, i \in I\right\}$. Then

$$
g_{i}\left(y_{\sigma}\right)=\frac{\left(x_{i} \partial f / \partial x_{i}\right) \circ \pi_{\sigma}\left(y_{\sigma}\right)}{\prod_{j=1}^{n} y_{\sigma, j}^{l\left(a^{j}(\sigma)\right)}}
$$

and so

$$
\left(g_{i} \mid E_{\sigma, I}\right)\left(y_{\sigma}\right)=\frac{\left(x_{i} \partial f_{\gamma} / \partial x_{i}\right) \circ \pi_{\sigma}\left(y_{\sigma}\right)}{\prod_{j \notin I} y_{\sigma, j}^{l\left(a^{j}(\sigma)\right)}} .
$$

Note that $\pi_{\sigma} \mid\left(K^{*}\right)^{n}$ is bijective. Put $y^{0}=\left(y_{1}^{0}, y_{2}^{0}, \ldots, y_{n}^{0}\right)=\pi_{\sigma}^{-1}\left(x^{0}\right)$ and

$$
\tilde{y}_{j}^{0}:= \begin{cases}y_{j}^{0} & \text { if } j \notin I, \\ 0 & \text { if } j \in I .\end{cases}
$$

Then

$$
g_{i}\left(\tilde{y}^{0}\right)=\frac{\left(x_{i} \partial f_{\gamma} / \partial x_{i}\right) \circ \pi_{\sigma}\left(y^{0}\right)}{\prod_{j=1}^{n}\left(y_{j}^{0}\right)^{l\left(a^{j}(\sigma)\right)}}=0
$$

for $1 \leq i \leq n$.

On the other hand, $\pi_{\sigma}\left(E_{\sigma, I}\right)=0$ by Lemma 3.7 in [2, p. 471]. So $\sum_{i=1}^{n}\left|g_{i}\left(\tilde{y}_{0}\right)\right|^{2}=0$ for $\tilde{y}^{0} \in E_{\sigma, I} \subset \pi_{\sigma}^{-1}(0) \subset \pi^{-1}(0)$. This completes the proof of the "if" part of (3.2).

(3.3) Lemma. If $f(x)$ is nondegenerate, then the following four statements are equivalent to each other for each $k=\left(k_{1}, k_{2}, \ldots, k_{n}\right) \in \mathbf{Z}_{+}^{n}$. 
(3.3.1) The $n$-tuple $k$ is an element of $\Lambda_{+}(f)$.

(3.3.2) The inequality

$$
l\left(a^{i}(\sigma)\right) \leq\left\langle k, a^{i}(\sigma)\right\rangle
$$

holds for any $i, 1 \leq i \leq n$, and any $n$-dimensional simplex $\sigma \in \Sigma$.

(3.3.3) Let $b^{i}, 1 \leq i \leq m$, be the all vectors of $\mathbf{Z}_{+}^{n *}$ such that $\mathbf{R}_{+} b^{i}$ is a one-dimensional cone of $\Gamma^{*}(f)$, namely $\gamma\left(b^{i}\right)$ is an $(n-1)$-dimensional face of $\Gamma_{+}(f)$. Then the inequality

$$
l\left(b^{i}\right) \leq\left\langle k, b^{i}\right\rangle
$$

holds for any $i, 1 \leq i \leq m$.

(3.3.4) The $n$-tuple $k$ is an element of $\Gamma_{+}(f)$.

Proof. It is clear that for any $n$-dimensional simplex $\sigma \in \Sigma$,

$$
|\operatorname{Grad} f| \circ \pi_{\sigma}\left(y_{\sigma}\right)=\left|y_{1}^{l\left(a^{1}(\sigma)\right)} y_{2}^{l\left(a^{2}(\sigma)\right)} \cdots y_{n}^{l\left(a^{n}(\sigma)\right)}\right|\left(\sum_{i=1}^{n}\left|g_{i}\left(y_{\sigma}\right)\right|^{2}\right)^{1 / 2}
$$

and

$$
\left|x^{k}\right| \circ \pi_{\sigma}\left(y_{\sigma}\right)=\prod_{i=1}^{n}\left|x_{i}^{k_{i}}\right| \circ \pi_{\sigma}\left(y_{\sigma}\right)=\left|y_{1}^{K_{1}} y_{2}^{K_{2}} \cdots y_{n}^{K_{n}}\right|
$$

where $K_{i}=\sum_{j=1}^{n} k_{j} a_{j}^{i}(\sigma)=\left\langle k, a^{i}(\sigma)\right\rangle$.

This implies that (3.3.1) and (3.3.2) are equivalent to each other by (3.2).

Since $\left\{b^{i} \mid 1 \leq i \leq m\right\}$ is a subset of $\left\{a^{i}(\sigma) \mid 1 \leq i \leq n\right.$, all $n$-dimensional simplices $\sigma$ of $\Sigma$, it is clear that (3.3.3) follows from (3.3.2).

Since $\Sigma$ is a subdivision of $\Gamma^{*}(g)$, for any $n$-dimensional simplex $\sigma$ there is an $n$-dimensional cone $\sigma\left(\Gamma^{*}\right)$ of $\Gamma^{*}(f)$ which contains $\sigma$. Then $\sigma\left(\Gamma^{*}\right)$ is a linear combination of $b^{i}, 1 \leq i \leq m$, with coefficient $\mathbf{R}_{+}$. We may assume that

$$
\sigma\left(\Gamma^{*}\right)=\mathbf{R}_{+} b^{1}+\mathbf{R}_{+} b^{2}+\cdots+\mathbf{R}_{+} b^{r}
$$

for simplicity of notation. Then $\bigcap_{i=1}^{r} \gamma\left(b^{i}\right)=\bigcap_{i=1}^{n} \gamma\left(a^{i}(\sigma)\right)$ is a one-point set. Let $p=\left(p_{1}, p_{2}, \ldots, p_{n}\right) \in \Gamma_{+}(f)$ be the point. There are nonnegatives $s_{i j}$, $1 \leq i \leq n, 1 \leq j \leq r$, such that

$$
a^{i}(\sigma)=\sum_{j=1}^{r} s_{i j} b^{j} .
$$

Now we have the equation

$$
l\left(a^{i}(\sigma)\right)=\left\langle p, a^{i}(\sigma)\right\rangle=\sum_{j=1}^{r} s_{i j}\left\langle p, b^{j}\right\rangle=\sum_{j=1}^{r} s_{i j} l\left(b^{j}\right) .
$$

Suppose that (3.3.3) is true; then

$$
l\left(a^{i}(\sigma)\right)=\sum_{j=1}^{r} s_{i j} l\left(b^{j}\right) \leq \sum_{j=1}^{r} s_{i j}\left\langle k, b^{j}\right\rangle=\left\langle k, \sum_{j=1}^{r} s_{i j} b^{j}\right\rangle=\left\langle k, a^{i}(\sigma)\right\rangle .
$$

So (3.3.2) is proved. 
It is clear that $l\left(b^{i}\right) \leq\left\langle k, b^{i}\right\rangle$ for $1 \leq i \leq m$ if and only if $k \in \Gamma_{+}(f)$. So (3.3.3) and (3.3.4) are equivalent to each other. This completes the proof of (3.3).

The "only if" part of (1.7) is an immediate corollary of (3.3).

Proof of "if" of (1.7). Let $\sigma$ be an $n$-dimensional simplex of $\Sigma$ and $\{p=$ $\left.\left(p_{1}, p_{2}, \ldots, p_{n}\right)\right\}=\bigcap_{i=1}^{n} \gamma\left(a^{i}(\sigma)\right)$, a vertex of $\Gamma_{+}(f)$. By the hypothesis: $\Lambda_{+}(f)$ $=\Gamma_{+}(f), p \in \Lambda_{+}(f)$, namely

$$
|\operatorname{Grad} f| \geq \varepsilon\left|x^{p}\right|
$$

in a neighborhood of the origin of $K^{n}$ for some positive $\varepsilon$. So we have

$$
\begin{gathered}
|\operatorname{Grad} f| \circ \pi_{\sigma}\left(y_{\sigma}\right)=\left|y_{1}^{l\left(a^{1}(\sigma)\right)} y_{2}^{l\left(a^{2}(\sigma)\right)} \cdots y_{n}^{l\left(a^{n}(\sigma)\right)}\right|\left(\sum_{i=1}^{n}\left|g_{i}\left(y_{\sigma}\right)\right|^{2}\right)^{1 / 2} \\
\geq \varepsilon\left|x^{p}\right| \circ \pi_{\sigma}\left(y_{\sigma}\right)=\varepsilon \prod_{i=1}^{n}\left|x_{i}^{p_{i}}\right| \circ \pi_{\sigma}\left(y_{\sigma}\right)=\varepsilon\left|y_{1}^{K_{1}} y_{2}^{K_{2}} \cdots y_{n}^{K_{n}}\right|
\end{gathered}
$$

where $K_{i}=\sum_{j=1}^{n} p_{j} a_{j}^{i}(\sigma)=\left\langle p, a^{i}(\sigma)\right\rangle=l\left(a^{i}(\sigma)\right)$.

So $\sum_{i=1}^{n}\left|g_{i}\right|^{2}>0$ in a neighborhood of $\pi_{\sigma}^{-1}(0)$, and by (3.2) the germ $f$ is nondegenerate. This completes the proof of the "if" of (1.7).

This completes the proof of (1.7).

(3.4) Corollary. If $f(x) \in A\left(K^{n}\right)$ is nondegenerate and $V(f)=\{0\}$. Then the family $F(x ; u):=f(x)+u g(x)$ is topologically trivial along $I=[0, \delta]$ for any $g(x) \in A\left(K^{n}\right)$ with $\Gamma_{+}(g) \subset \operatorname{Int} \Gamma_{+}(f)$ where $\delta$ is positive and sufficiently small.

Proof. This follows immediately from (1.6) and (1.7).

Acknowledgment. I would like to thank Toshizumi Fukui, who pointed out a mistake in the previous version of this paper, and the referee for useful suggestions.

\section{REFERENCES}

1. S. H. Chang and Y. C. Lu, On $C^{0}$-sufficiency of complex jets, Canad. J. Math. 25 (1973), 874-880.

2. T. Fukui and E. Yoshinaga, The modified analytic trivialization of families of real analytic functions, Invent. Math. 82 (1985), 467-477.

3. N. H. Kuiper, $C^{1}$-equivalence of functions near isolated critical point, Symposium Infinite Dimensional Topology, Ann. of Math. Studies, no. 69, Princeton Univ. Press, Princeton, N.J., 1972.

4. T. C. Kuo, On $C^{0}$-sufficiency of jets of potential functions, Topology 8 (1969), 167-171.

5. M. Suzuki, The stratum with constant Milnor number of a mini-transversal family of a quasihomogeneous function of corank two, Topology 23 (1983), 101-115. 
6. A. N. Varchenko, Newton polyhedra and estimation of oscillating integrals, Functional Anal. Appl. 10 (1977), 175-196.

7. E. Yoshinaga, The modified analytic trivialization of real analytic families via blowing-ups, J. Math. Soc. Japan 40 (1988), 161-180.

Department of Mathematics, Faculty of Education, Yokohama National University, YOKOHAMA, JAPAN 